\title{
Molecular Mechanisms of Trehalose in Modulating Glucose Homeostasis in Diabetes
}

\author{
Running Title: Trehalose and Glucose Homeostasis \\ Yaribeygi, Habib; Yaribeygi, Alijan; Sathyapalan, Thozhukat; \\ Sahebkar, Amirhossein
}

\begin{abstract}
Diabetes mellitus is the most prevalent metabolic disorder contributing to significant morbidity and mortality in humans. Many preventative and therapeutic agents have been developed for normalizing glycemic profile in patients with diabetes. In addition to various pharmacologic strategies, many non-pharmacological agents have also been suggested to improve glycemic control in patients with diabetes. Trehalose is a naturally occurring disaccharide which is not synthesized in human but is widely used in food industries. Some studies have provided evidence indicating that it can potentially modulate glucose metabolism and help to stabilize glucose homeostasis in patients with diabetes. Studies have shown that trehalose can significantly modulate insulin sensitivity via at least 7 molecular pathways leading to better control of hyperglycemia. In the current study, we concluded about possible anti-hyperglycemic effects of trehalose suggesting trehalose as a potentially potent nonpharmacological agent for the management of diabetes.
\end{abstract}

Keywords: Diabetes Mellitus, Trehalose, Oxidative Stress, Glucose, Homeostasis.

This accepted manuscript of a work published in Diabetes and Metabolic Syndrome: Clinical research and reviews is licensed under a Creative Commons Attribution-NonCommercialNoDerivatives 4.0 International License. 


\section{Introduction}

The global prevalence of diabetes mellitus (DM) is rising exponentially [1]. This chronic disorder has paramount effects on various metabolic pathways as well as on the physiologic function of most organs and hence responsible for a wide variety of diabetesinduced tissue dysfunctions known as diabetic complications [2, 3]. DM acts as a potent upstream event for many pathophysiologic mechanisms such as oxidative stress, inflammation, apoptosis and fibrosis which in turn induce the onset and progression of various forms of diabetic complications [4,5]. Diabetes and its related complications impose a significant economic burden to the health systems and are responsible for many cases of disabilities and death in human [6, 7]. Therefore, normalizing glycemic profile and management of chronic hyperglycemia by pharmacological and non-pharmacological approaches are of paramount importance [8-10].

Trehalose is a disaccharide carbohydrate which is synthesized in many organisms ranging from bacteria to plants [11]. Although this natural sweetener molecule could not be synthesized in humans, it has some biological roles and effects on various metabolic pathways after consumption [12-14]. It is used extensively in the food industry, biotechnology and pharmaceutical industry. There is some evidence suggesting that it can potentially modulate glucose homeostasis $[15,16]$. In the current review, we assessed the possible links between trehalose usage and DM in humans and whether it can modulate hyperglycemia toward the physiologic state.

\section{Trehalose, Biochemistry and Biology}

Trehalose is a naturally-occurring, non-reducing sugar formed by two D-glucose molecules linked by $\alpha-1,1$-glycosidic bindings [17, 18]. This unreactive disaccharide is synthesized by a wide variety of species ranging from prokaryotes, fungi, invertebrates, insects, plants, and yeast and has significant metabolic roles including storage of energy [18]. The synthetic form of Trehalose was produced by enzymatic processes for the first time in 1995 [19], and at present is extensively used in food industries as a stabilizer for protecting foods against dryness, freezing and osmotic pressure stresses [16]. In comparison with sucrose, trehalose has lower sweetener potency and is broken into two glucose molecules by the enzyme trehalase and provides a high amount of energy $[18,20]$.

It also acts as an osmo-protectant especially against ethanol and osmotic pressures. It provides a stabilizing effect for the cellular membrane by increasing the plasma membrane tolerance to dehydration, shriveling and temperature shock [21]. It has suggested that nonreactivity and high stability properties of trehalose are due to the very low energy of the glycoside oxygen bindings to its two hexose rings [21]. Although trehalase enzyme is widely expressed in human and founded in the brush border of mucosal intestinal cells, no evidence exists about the trehalose production in human [18].

\section{Molecular Mechanisms Involved in Insulin Resistance}

Insulin resistance; which is a key event in the development of dysmetabolic states such as non-alcoholic fatty liver disease, DM and metabolic syndrome; is a complicated condition 
involving several molecular mechanisms [22, 23]. In a previous review we found that at least 10 molecular pathways are included in peripheral resistance to insulin including reduced response to circulatory insulin such as up-regulation of PTP1B (protein-tyrosine phosphatase 1B), inflammatory mediators and adipokines secretion, oxidative stress, ER (endoplasmic reticulum) stress, lower IRS-1 (insulin receptor substrates-1) phosphorylation, adipocytes metabolism, augmented insulin degradation, defect in GLUT-4 (glucose transporter-4) activity, lower capacity of receptors to binding to insulin and mitochondrial dysfunction [23]. As a result, any pharmacologic or non-pharmacological agents which modulate these molecular mechanisms may be also able to regulate insulin sensitivity [8, 23-25].

\section{Possible Links between Trehalose and Diabetes Mellitus}

Some recent evidence have shown that trehalose can potentially modulate glucose homeostasis $[15,16]$. In following sections, we examine these potentials of trehalose (table 1).

\section{Trehalose and Insulin Sensitivity}

Trehalose can potentially increase insulin sensitivity and improve insulin resistance in diabetic milieu [26]. This effect is exerted either directly on glucose signaling pathways or indirectly via alleviating pathophysiologic pathways such as oxidative stress, inflammation and/or improvement in lipid metabolism [26]. Arai et al in 2010 demonstrated that trehalose induces insulin sensitivity by several mechanisms including a reduction in inflammatory mediators such as MCP-1 (monocyte chemotactic protein-1), TNF- $\alpha$ (tumor necrosis factoralpha), and PAI-1 (plasminogen activator inhibitor-1) and improvement in adipocytes function by inhibition of lipid accumulation [26]. Arai and coworkers in 2013 presented further evidence indicating that 8 weeks trehalose consumption mitigates insulin resistance by adiponectin secretion, lipid profile correction and up-regulation of the IRS-1 and IRS-2 mRNA expression in obese mice [27]. Mizote and colleagues in 2016 found in a clinical trial that trehalose improved insulin resistance by adiponectin release and PAI-1 down-regulation in people at risk of metabolic syndrome [15]. Higgins et al in 2018 showed that trehalose is a potent analog for ALOXE3 (Epidermis-type Lipoxygenase 3) which in turn induces insulin sensitivity via PPAR- $\gamma$ (peroxisome proliferator-activated receptor gamma) dependent mechanism in mice [28]. These evidence strongly suggest that trehalose induces insulin sensitivity in hyperlipidemia or in the hyperglycemic milieu.

\section{Trehalose and Postprandial Glucose/Insulin Secretion}

Reducing insulin secretion is the most known effects of trehalose on glucose homeostasis and is supported by various studies [26, 29, 30]. Arai and coworkers in 2010 observed that trehalose markedly reduced the fasting insulin levels in comparison with glucose and maltose in rats [26]. Oku et al in 2000 reported that trehalose intake has remarkable effects on blood glucose in people with higher levels of trehalase activity than people with low trehalase activity. Trehalose had a potent inhibitory effect on insulin release in these subjects [29]. Also, Maki et al in 2009 reported that trehalose intake in obese men elicited lower postprandial insulin secretory response than to that of glucose [30]. Yoshizane and colleagues in 2017 observed in healthy volunteers that trehalose ingestion did not induce a rapid rise in postprandial glucose and did not stimulate GIP (gastric inhibitory protein) secretion [16]. These 
evidence clearly confirm that trehalose has weaker stimulatory effects on postprandial insulin release in comparison with other sugars [26, 30]. It has also been suggested that the trehalase acts in a manner dependent on insulin level demonstrating the intricate relationship between insulin levels and trehalase activity [31]. These finding also showed that trehalose reduces insulin dependent postprandial adipocyte accumulation which is regularly seen after carbohydrate consumption [16, 26].

\section{Trehalose and Glucose Metabolism}

Trehalose has shown to normalize glucose metabolic pathways [32, 33]. Sato et al in 1999 demonstrated that animals consuming parenteral trehalose have improved glucose metabolism and nutritional indices than after consuming other disaccharides [32]. Also, van Can and coworkers in 2012 provided data indicating trehalose improves glucose metabolism in patients with glucose intolerance [34]. Yasugi et al in 2017 suggested that animals using trehalose as nutrient have improved metabolism which is gained via adaptation [35]. These evidence implied that trehalose may directly modulate glucose metabolism but the exact mechanisms need to be elucidated in further studies.

\section{Trehalose and Lipid Metabolism}

Correction of the lipid profile may be one of the other possible mechanisms by which trehalose improves glucose homeostasis [26]. There is growing evidence that trehalose directly modulates secretion of adipokines and increases adiponectin release leading to improvement in lipid metabolism [15, 27]. Since sub-optimal lipid profile is closely linked to insulin resistance disorders as well as DM; we suggest that any improvement in lipid metabolism could also effectively improve insulin sensitivity [15].

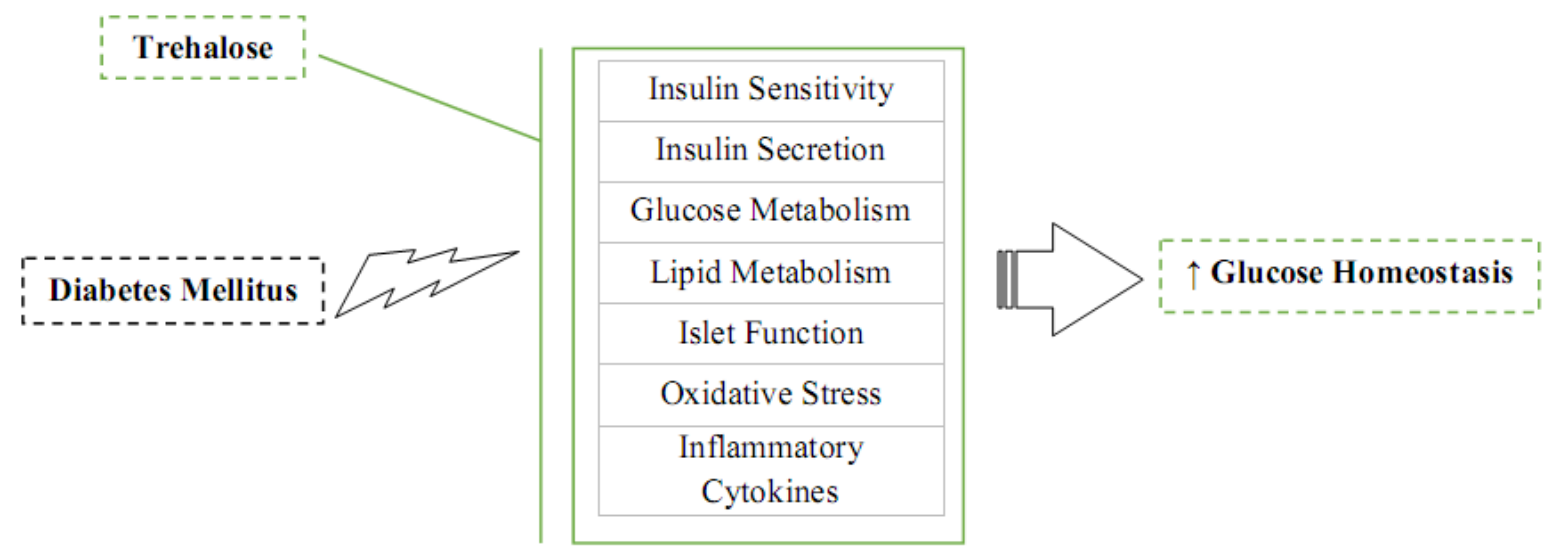

Figure 1; Molecular mechanisms by which trehalose prevents and reverses the effects of DM on glucose homeostasis

\section{Trehalose and Improvement in Islet Function}

Trehalose consumption could improve pancreatic islet function in several ways [36, 37]. Emerging evidence indicates that trehalose prevents apoptosis and autophagy processes, both of which are involved in beta cell dysfunction [36-38]. Beattie and coworkers in 1997 provided first direct evidence suggesting long-term trehalose consumption improves pancreatic 
beta cells' efficiency in patients with diabetes [37]. Pan et al in 2018 demonstrated that trehalose is able to suppress apoptosis in diabetic animals [38]. Lin et al in 2016 established an experimental study confirming trehalose diminishes pathogenic mechanisms such as apoptosis, pyroptosis and autophagy probably by its anti-oxidant and anti-inflammatory potentials in pancreatic cells of diabetic rats [39]. This beneficial effect was previously suggested by Xu and coworkers in 2013 in neuronal cells where they showed that trehalose improved diabetesinduced neuronal defects by correcting the autophagy process [40]. Chen and colleagues in 2016 reported that lower concentration of trehalose markedly improves islet function by ameliorating islet amyloid polypeptide synthesis in patients with diabetes [36]. These data strongly suggest that trehalose consumption may improve islet function and glucose homeostasis.

\section{Trehalose and Oxidative Stress}

Free radical overload and oxidative stress occur in patients with diabetes [41]. Metabolic derangements which develop during DM markedly induces free radical generation which in turn results in an increase in peripheral resistance to insulin [32, 41]. Some experiments suggest that trehalose can potentially reduce oxidative stress [42]. Mizunoe and colleagues in 2018 showed that trehalose protects against oxidative damages by Nrf2 (nuclear factor erythroid 2-related factor 2; a nuclear factor regulates antioxidant elements expression)dependent antioxidant elements up-regulation in cell lines of mouse hepatocytes [42]. AlvarezPeral et al in 2002 demonstrated that trehalose has a protective role against oxidative stress in Candida albicans [43]. Moreover, Tang et al in 2017 reported that trehalose diminished mitochondria-induced oxidative stress by regulation of the autophagy process in patients with osteoarthritis [44]. Echigo et al in 2012 found that trehalose improved oxidative damages in the brain after subarachnoid bleeding in experimental studies, both in vitro and in vivo [45]. These data in addition to other evidence demonstrates potent antioxidative effects of trehalose which contributes to the potential antihyperglycaemic effects of this non-reducing disaccharide [46].

\begin{tabular}{|c|c|c|}
\hline Molecular Pathway & Influence of Trehalose & References \\
\hline Insulin Sensitivity & $\begin{array}{c}\text { Improves insulin sensitivity by several mechanisms } \\
\text { including IRS up-regulation, PPAR- } \gamma \text { dependent } \\
\text { mechanism and ameliorating other pathophysiologic } \\
\text { pathways }\end{array}$ & {$[26-28]$} \\
\hline Insulin Secretion & Reduces postprandial insulin release & {$[26,29,30]$} \\
\hline Glucose Metabolism & $\begin{array}{c}\text { Improves glucose metabolism and regulates } \\
\text { postprandial glucose levels }\end{array}$ & {$[32-35]$} \\
\hline
\end{tabular}




\begin{tabular}{|c|c|c|}
\hline Lipid Metabolism & $\begin{array}{c}\text { Corrects lipid metabolism by modulating } \\
\text { postprandial insulin release }\end{array}$ & {$[15,26,27]$} \\
\hline Islet Function & Improves beta cell function & {$[36-40]$} \\
\hline $\begin{array}{c}\text { Oxidative Stress } \\
\text { Inflammatory } \\
\text { Cytokines }\end{array}$ & $\begin{array}{c}\text { Attenuates oxidative stress leading to improved } \\
\text { insulin sensitivity }\end{array}$ & {$[42-46]$} \\
\hline
\end{tabular}

Table 1; Various influences of trehalose on DM, trehalose consumption can modulate glucose homeostasis via at least 7 molecular mechanisms (IRSs=insulin receptor substrates, PPAR- $\gamma=$ Peroxisome proliferatoractivated receptor gamma)

\section{Trehalose and Inflammatory Cytokines}

Inflammatory mediators have significant roles in the pathophysiology of many complications of DM [49, 50]. It has been well established that the expression/release of inflammatory mediators is up-regulated in patients with insulin resistance [4, 51]. There is growing evidence that trehalose inhibits inflammatory responses by ameliorating inflammatory mediators [26, 47]. Consumption of trehalose markedly reduced inflammatory cytokines such as TNF- $\alpha$, MCP-1 and PAI-1 in experimental studies [26, 48]. In addition, Echigo et al in 2012 showed that trehalose ameliorated the inflammation in in-vitro and in-vivo models of subarachnoid hemorrhage [45]. This suggests that intake of trehalose improves insulin sensitivity at least partly via attenuating inflammatory responses [48].

\section{Other Possible Links}

There is some evidence suggesting other potential relationships between trehalose consumption and insulin sensitivity such as thermogenesis [52], stabilizing insulin [53], and improvement in mitochondrial dysfunction [44]. But these molecular pathways need further investigation by in vivo and in vitro studies.

Trehalose has been shown to inhibit multiple glucose transporter (GLUT) receptors through binding in the inward open conformation of these receptors [54, 55]. By inhibiting GLUT function trehalose could potentially prevent excess glucose absorption in the intestine. It has also reported that trehalose reduces postprandial glycemic excursions in patients with impaired glucose tolerance [56]. This suggests that ingestion of trehalose with daily meals could potentially prevent hyperglycemia and development of diabetes. 


\section{Conclusion}

Trehalose is a non-reducing carbohydrate widely produced by different species but not in the human. It has lower sweetener potency than sucrose and is broken down into two molecules of glucose by trehalase enzyme activity. This enzyme is expressed in humans and is localized in the brush border of endothelial cells of the intestine. In addition to providing energy in other species, trehalose can modulate glucose homeostasis by at least 7 molecular pathways. Data suggests that trehalose consumption improve hyperglycemic milieu by ameliorating pathophysilogical mechanisms such as oxidative stress, inflammation and apoptosis, improving beta cell function, reducing postprandial insulin release and normalizing lipid profile. These molecular mechanisms suggest the role of trehalose as a potential nonpharmacological agent for the management of glycaemia in patients with diabetes, however, more clinical trials are needed to assess this.

\section{Acknowledgment}

The authors are thankful to the Clinical Research and Development Unit of the Baqiyatallah Hospital (Tehran, Iran).

\section{Conflict of Interests}

The authors clearly declare that have no conflict of interest in this study.

\section{References}

Mayer-Davis, E.J., et al., Incidence trends of type 1 and type 2 diabetes among youths, 2002-

2012. New England Journal of Medicine, 2017. 376(15): p. 1419-1429.

Yaribeygi, $\mathrm{H}_{\text {., }}$ et al., Antioxidative potential of antidiabetic agents: A possible protective

mechanism against vascular complications in diabetic patients. Journal of cellular physiology, 2019. 234(3): p. 2436-2446.

Abraham, T.M., et al., Trends in diabetes incidence: the Framingham Heart Study. Diabetes care, 2015. 38(3): p. 482-487.

Yaribeygi, H., et al., A review of the anti-inflammatory properties of antidiabetic agents

4. providing protective effects against vascular complications in diabetes. Journal of cellular physiology, 2018.

Yaribeygi, H., A.E. Butler, and A. Sahebkar, Aerobic exercise can modulate the underlying mechanisms involved in the development of diabetic complications. Journal of cellular physiology, 2019.

Association, A.D., Economic costs of diabetes in the US in 2017. Diabetes Care, 2018. 41(5):

p. 917-928.

Rosella, L., et al., Impact of diabetes on healthcare costs in a population-based cohort: a cost analysis. Diabetic Medicine, 2016. 33(3): p. 395-403.

Yaribeygi, H., et al., Antidiabetic potential of saffron and its active constituents. Journal of cellular physiology, 2018.

Yaribeygi, H., et al., Molecular mechanisms by which aerobic exercise induces insulin sensitivity. Journal of cellular physiology, 2019. 
Elbein, A.D., et al., New insights on trehalose: a multifunctional molecule. Glycobiology,

2003. 13(4): p. 17R-27R.

Figueroa, C.M., et al., Trehalose 6-phosphate coordinates organic and amino acid

12.

metabolism with carbon availability. The Plant Journal, 2016. 85(3): p. 410-423.

Eleutherio, E., et al., Revisiting yeast trehalose metabolism. Current genetics, 2015. 61(3): p.

13.

263-274.

Wang, X., Y. Du, and D. Yu, Trehalose phosphate synthase 5-dependent trehalose

metabolism modulates basal defense responses in Arabidopsis thaliana. Journal of

integrative plant biology, 2018.

Mizote, A., et al., Daily intake of trehalose is effective in the prevention of lifestyle-related

diseases in individuals with risk factors for metabolic syndrome. Journal of nutritional science

and vitaminology, 2016. 62(6): p. 380-387.

Yoshizane, C., et al., Glycemic, insulinemic and incretin responses after oral trehalose

ingestion in healthy subjects. Nutrition journal, 2017. 16(1): p. 9.

Thammahong, A., et al., Central role of the trehalose biosynthesis pathway in the 17.

pathogenesis of human fungal infections: opportunities and challenges for therapeutic

development. Microbiology and Molecular Biology Reviews, 2017. 81(2): p. e00053-16.

Feofilova, E., et al., Trehalose: chemical structure, biological functions, and practical

application. Microbiology, 2014. 83(3): p. 184-194.

Sugimoto, T., Production of trehalose by starch-glycation method with enzymes and its use.

Food Industry, 1995. 38: p. 34-39.

Moriano, M.E. and C. Alamprese, Honey, trehalose and erythritol as sucrose-alternative

20.

sweeteners for artisanal ice cream. A pilot study. LWT-Food Science and Technology, 2017.

75: p. 329-334.

Schiraldi, C., I. Di Lernia, and M. De Rosa, Trehalose production: exploiting novel approaches.

Trends in biotechnology, 2002. 20(10): p. 420-425.

Freeman, A.M. and N. Pennings, Insulin resistance, in StatPearls [Internet]. 2018, StatPearls

Publishing.

Yaribeygi, H., et al., Insulin resistance: Review of the underlying molecular mechanisms.

23.

Journal of cellular physiology, 2018.

Yaribeygi, H., et al., MicroRNAs and type 2 diabetes mellitus: Molecular mechanisms and the

effect of antidiabetic drug treatment. Metabolism, 2018.

Yaribeygi, H., S.L. Atkin, and A. Sahebkar, Mitochondrial dysfunction in diabetes and the

25.

regulatory roles of antidiabetic agents on the mitochondrial function. Journal of cellular

physiology, 2018.

Arai, C., et al., Trehalose prevents adipocyte hypertrophy and mitigates insulin resistance.

Nutrition research, 2010. 30(12): p. 840-848.

Arai, C., et al., Trehalose prevents adipocyte hypertrophy and mitigates insulin resistance in

19.

mice with established obesity. Journal of nutritional science and vitaminology, 2013. 59(5): p.

393-401.

Higgins, C.B., et al., Hepatocyte ALOXE3 is induced during adaptive fasting and enhances

28.

insulin sensitivity by activating hepatic PPARY. JCl insight, 2018. 3(16).

$\mathrm{Oku}, \mathrm{T}$. and S. Nakamura, Estimation of intestinal trehalase activity from a laxative threshold

of trehalose and lactulose on healthy female subjects. European journal of clinical nutrition, 2000. 54(10): p. 783.

Maki, K.C., et al., Acute effects of low insulinemic sweeteners on postprandial insulin and

30.

glucose concentrations in obese men. International journal of food sciences and nutrition, 2009. 60(sup3): p. 48-55.

Richards, A., et al., Trehalose: a review of properties, history of use and human tolerance, and results of multiple safety studies. Food and chemical toxicology, 2002. 40(7): p. 871-898. 
Sato, S., et al., Trehalose can be used as a parenteral saccharide source in rabbits. The Journal of nutrition, 1999. 129(1): p. 158-164.

Seo, Y., et al., Metabolic shift from glycogen to trehalose promotes lifespan and healthspan

in Caenorhabditis elegans. Proceedings of the National Academy of Sciences, 2018. 115(12):

p. E2791-E2800.

van Can, J.G., et al., Reduced glycaemic and insulinaemic responses following trehalose and isomaltulose ingestion: implications for postprandial substrate use in impaired glucosetolerant subjects. British Journal of Nutrition, 2012. 108(7): p. 1210-1217.

Yasugi, T., T. Yamada, and T. Nishimura, Adaptation to dietary conditions by trehalose

metabolism in Drosophila. Scientific Reports, 2017. 7(1): p. 1619.

Chen, C.-H., et al., Influence of trehalose on human islet amyloid polypeptide fibrillation and aggregation. RSC Advances, 2016. 6(18): p. 15240-15246.

Beattie, G.M., et al., Trehalose: a cryoprotectant that enhances recovery and preserves

function of human pancreatic islets after long-term storage. Diabetes, 1997. 46(3): p. 519523.

Pan, H., et al., Trehalose prevents sciatic nerve damage to and apoptosis of Schwann cells of streptozotocin-induced diabetic C57BL/6J mice. Biomedicine \& Pharmacotherapy, 2018. 105: p. 907-914.

Lin, C.-F., et al., Quercetin-rich Guava (Psidium guajava) juice in combination with trehalose reduces autophagy, apoptosis and pyroptosis formation in the kidney and pancreas of type II diabetic rats. Molecules, 2016. 21(3): p. 334.

$\mathrm{Xu}, \mathrm{C}$. , et al., Trehalose prevents neural tube defects by correcting maternal diabetes- $\quad 40$. suppressed autophagy and neurogenesis. American Journal of Physiology-Endocrinology and Metabolism, 2013. 305(5): p. E667-E678.

Yaribeygi, H., S.L. Atkin, and A. Sahebkar, A review of the molecular mechanisms of 41. hyperglycemia-induced free radical generation leading to oxidative stress. Journal of cellular physiology, 2019. 234(2): p. 1300-1312.

Mizunoe, Y., et al., Trehalose protects against oxidative stress by regulating the Keap1-Nrf2 and autophagy pathways. Redox biology, 2018. 15: p. 115-124.

Alvarez-Peral, F.J., et al., Protective role of trehalose during severe oxidative stress caused by hydrogen peroxide and the adaptive oxidative stress response in Candida albicans.

Microbiology, 2002. 148(8): p. 2599-2606.

Tang, Q., et al., Trehalose ameliorates oxidative stress-mediated mitochondrial dysfunction and ER stress via selective autophagy stimulation and autophagic flux restoration in osteoarthritis development. Cell death \& disease, 2017. 8(10): p. e3081.

Echigo, R., et al., Trehalose treatment suppresses inflammation, oxidative stress, and 45. vasospasm induced by experimental subarachnoid hemorrhage. Journal of translational medicine, 2012. 10(1): p. 80.

$\mathrm{Hu}, \mathrm{J} .-\mathrm{H} .$, et al., Effects of trehalose supplementation on semen quality and oxidative stress variables in frozen-thawed bovine semen. Journal of animal Science, 2010. 88(5): p. 16571662.

Arai, C., et al., Trehalose suppresses lipopolysaccharide-induced osteoclastogenesis bone marrow in mice. Nutrition Research, 2001. 21(7): p. 993-999.

Taya, K., K. Hirose, and S. Hamada, Trehalose inhibits inflammatory cytokine production by protecting IKB- $\alpha$ reduction in mouse peritoneal macrophages. Archives of oral biology, 2009. 54(8): p. 749-756.

Yaribeygi, H., et al., Effects of antidiabetic drugs on NLRP3 inflammasome activity, with a focus on diabetic kidneys. Drug discovery today, 2018.

Yaribeygi, H., S.L. Atkin, and A. Sahebkar, Interleukin-18 and diabetic nephropathy: A review. Journal of cellular physiology, 2018. 
Yaribeygi, H., et al., Crocin improves renal function by declining Nox-4, IL-18, and $p 53$ expression levels in an experimental model of diabetic nephropathy. Journal of cellular biochemistry, 2018. 119(7): p. 6080-6093.

Zhang, Y., et al., TFEB-dependent induction of thermogenesis by the hepatocyte SLC2A inhibitor trehalose. Autophagy, 2018. 14(11): p. 1959-1975.

Mansfield, K.M. and H.D. Maynard, Site-Specific Insulin-Trehalose Glycopolymer Conjugate by Grafting from Strategy Improves Bioactivity. ACS Macro Letters, 2018. 7(3): p. 324-329. Mayer, A.L., et al., SLC2A8 (GLUT8) is a mammalian trehalose transporter required for trehalose-induced autophagy. Sci Rep, 2016. 6: p. 38586.

DeBosch, B.J., et al., Trehalose inhibits solute carrier 2A (SLC2A) proteins to induce autophagy and prevent hepatic steatosis. Sci Signal, 2016. 9(416): p. ra21.

van Can, J.G., et al., Reduced glycaemic and insulinaemic responses following trehalose and isomaltulose ingestion: implications for postprandial substrate use in impaired glucosetolerant subjects. Br J Nutr, 2012. 108(7): p. 1210-7. 